EPHOU-17-017

December 2017

\title{
A Bulk Localized State and New Holographic Renormalization Group Flow in 3D Spin-3 Gravity
}

\author{
Ryuichi NAKAYAma*, and Tomotaka SuzukI ${ }^{\dagger}$ \\ Division of Physics, Graduate School of Science, \\ Hokkaido University, Sapporo 060-0810, Japan
}

\begin{abstract}
We construct a localized state of a scalar field in 3D spin-3 gravity. 3D spin-3 gravity is thought to be holographically dual to $\mathrm{W}_{3}$ extended CFT on a boundary at infinity. It is known that while $\mathrm{W}_{3}$ algebra is a non-linear algebra, in the limit of large central charge $c$ a linear finite-dimensional subalgebra generated by $W_{n}(n=0, \pm 1, \pm 2)$ and $L_{n}(n=0, \pm 1)$ is singled out. The localized state is constructed in terms of these generators. To write down an equation of motion for a scalar field which is satisfied by this localized state it is necessary to introduce new variables for an internal space $\alpha^{ \pm}, \beta^{ \pm}, \gamma$, in addition to ordinary coordinates $x^{ \pm}$and $y$. The higher-dimensional space, which combines the bulk spacetime with the 'internal space', which is an ana$\log$ of superspace in supersymmetric theory, is introduced. The 'physical bulk spacetime' is a 3D hypersurface with constant $\alpha^{ \pm}, \beta^{ \pm}$and $\gamma$ embedded in this space. We will work in Poincaré coordinates of AdS space and consider Wquasi-primary operators $\Phi_{\boldsymbol{h}}\left(x^{+}\right)$with a conformal weight $\boldsymbol{h}$ in the boundary and study two and three point functions of $\mathrm{W}$-quasi-primary operators transformed as $e^{i x^{+} L_{-1}^{h}} e^{\beta^{+} W_{-1}^{h}} \Phi_{\boldsymbol{h}}(0) e^{-\beta^{+} W_{-1}^{h}} e^{-i x^{+} L_{-1}^{h}}$. Here $L_{n}^{h}$ and $W_{n}^{h}$ are $\operatorname{sl}(3, \mathrm{R})$ generators in the hyperbolic basis for Poincaré coordinates. It is shown that in the $\beta^{+} \rightarrow \infty$ limit, the conformal weight changes to a new value $\boldsymbol{h}^{\prime}=\boldsymbol{h} / 2$. This may be regarded as a Renormalization Group (RG) flow. It is argued that this RG flow will be triggered by terms $\Delta S \propto \beta^{+} W_{-1}^{h}+\beta^{-} \bar{W}_{-1}^{h}$ added to the action.
\end{abstract}

\footnotetext{
*nakayama@particle.sci.hokudai.ac.jp

${ }^{\dagger}$ t-suzuki@particle.sci.hokudai.ac.jp
} 


\section{Introduction}

Higher spin theory is a toy model of string theory. In [1] 3d O $(\mathrm{N})$ vector theory is shown to be dual to $4 \mathrm{~d}$ Vasiliev theory 2]. In $3 \mathrm{~d}$ it is possible to truncate higher spins to $s \leq N$ and the dynamics of higher spin theory can be described in terms of $\operatorname{sl}(\mathrm{N}, \mathrm{R}) \oplus \operatorname{sl}(\mathrm{N}, \mathrm{R})$ Chern-Simons (CS) gauge theory. [3] In [4] [5] minimal model of higher spin theory was proposed by using coset construction. Black hole solution was obtained in [6].

We will focus on spin 3 gravity and $\mathrm{W}_{3}$ algebra. Spin-3 gravity in three dimensions can be formulated as $\mathrm{sl}(3, \mathrm{R}) \oplus \operatorname{sl}(3, \mathrm{R})$ Chern-Simons (CS) gauge theory. [10] 11$]$ This is considered to be dual to $\mathrm{W}_{3}$-extended $\mathrm{CFT}_{2}$ on the boundary. [3] The $\mathrm{W}_{3^{-}}$ extended conformal algebra is a non-linear algebra [7] [8] defined by

$$
\begin{aligned}
{\left[L_{m}, L_{n}\right]=} & (m-n) L_{m+n}+\frac{c}{12} m\left(m^{2}-1\right) \delta_{m+n, 0}, \\
{\left[L_{m}, W_{n}\right]=} & (2 m-n) W_{m+n}, \\
{\left[W_{m}, W_{n}\right]=} & -\frac{c}{36} m\left(m^{2}-1\right)\left(m^{2}-4\right) \delta_{m+n, 0} \\
& -\frac{1}{3}(m-n)\left(2 m^{2}+2 n^{2}-m n-8\right) L_{m+n} \\
& -10 \beta(m-n) \Lambda_{m+n}, \quad(m, n \in \mathbf{Z}) .
\end{aligned}
$$

Here $\Lambda_{m}$ is a normal-ordered operator

$$
\begin{aligned}
\Lambda_{m} & =\sum_{n=-\infty}^{\infty}\left(L_{m-n}, L_{n}\right)-\frac{3}{10}(m+3)(m+2) L_{m}, \\
& =\sum_{n \leq-2} L_{n} L_{m-n}+\sum_{n \geq-1} L_{m-n} L_{n}-\frac{3}{10}(m+3)(m+2) L_{m},
\end{aligned}
$$

and $\beta$ is a constant related to the central charge $c$ :

$$
\beta=\frac{16}{22+5 c} \text {. }
$$

The normalization of $W_{n}$ is modified compared to that in [8] by multiplying the right hand side of the equation for commutator $\left[W_{m}, W_{n}\right]$ by a factor -102 We will

\footnotetext{
${ }^{1}$ These Lie algebras are related to generalizations of $3 \mathrm{~d}$ diffeomorphism and local Lorentz transformations. They should not be confused with those associated with the chiral and anti-chiral $\mathrm{W}_{3}$ algebras on the $2 \mathrm{~d}$ boundary, which will be henceforth discussed in this paper.

${ }^{2}$ This makes the central charge term of this commutator negative for $c>0$. If we adopt a rule of hermitian conjugation, $\left(W_{n}\right)^{\dagger}=W_{-n}$, this creates negative norm states. Even if we define $\left(W_{n}\right)^{\dagger}=-W_{-n}$, there are negative norm states, since in the semi-classical limit the algebra gives $\left[W_{2}, W_{-2}\right]=-16 L_{0}$ and $\left[W_{1}, W_{-1}\right]=2 L_{0}$. See (1.4). This will be important, when applying the c-theorem 9 . In this paper this issue will not be studied.
} 
adopt the rule of hermitian conjugation $\left(W_{n}\right)^{\dagger}=W_{-n} 3$

In the semi-classical limit of the bulk theory $c \rightarrow \infty, \beta(1.3)$ is $\mathcal{O}\left(c^{-1}\right)$, and by dropping the $3 \mathrm{rd}$ term in (1.1), we can restrict discussion to the sub-algebra of the wedge modes, $L_{n}(n=0, \pm 1)$ and $W_{m}(m=0, \pm 1, \pm 2)$. It is a linear sl(3,R) algebra and given by

$$
\begin{aligned}
{\left[L_{m}, L_{n}\right] } & =(m-n) L_{m+n}, \quad\left[L_{m}, W_{n}\right]=(2 m-n) W_{m+n}, \\
{\left[W_{m}, W_{n}\right] } & =-\frac{1}{3}(m-n)\left\{2 m^{2}+2 n^{2}-m n-8\right\} L_{m+n} .
\end{aligned}
$$

While algebra $\operatorname{sl}(3, \mathrm{R}) \oplus \operatorname{sl}(3, \mathrm{R})$ is a generalization of diffeomorphism and local Lorentz symmetry in Chern-Simons (CS) gauge theory, this algebra $\mathrm{sl}(3, \mathrm{R}) \oplus \operatorname{sl}(3, \mathrm{R})$ is also a symmetry algebra of boundary CFT as well as the bulk, where each $\operatorname{sl}(3, \mathrm{R})$ is a global $\mathrm{W}_{3}$ algebra for rightmover and leftmover, respectively. In this paper we will address a new renormalization group flow in $\mathrm{W}_{3}$ confromal field theory (CFT) and its description in terms of a geometry of higher dimensional space for spin-3 gravity in $\mathrm{AdS}_{3}$ space, which is obtained by combining an 'internal space' and an ordinary spacetime. In CFT operators are assigned to each point in the space by moving by $L_{-1}$ and its anti-holomorphic partner $\bar{L}_{-1}$. In $\mathrm{W}_{3}$ extended CFT $W_{-2}$ and $W_{-1}$ also move operators in the 'internal space' 4

In $\mathrm{AdS}_{d+1}$ gravity the spacetime can be embedded as a hypersurface into a $\mathrm{d}+2$ dimensional Minkowski space $M^{d, 2}$. This flat space has $\mathrm{SO}(\mathrm{d}, 2)$ symmetry and at any point on the hypersurface we have an invariant subgroup $\mathrm{SO}(\mathrm{d}, 1)$. In the case of $\mathrm{AdS}_{3}, \mathrm{SO}(2,2)=\mathrm{SL}(2, \mathrm{R}) \otimes \mathrm{SL}(2, \mathrm{R})$ and the symmetry at a point in the bulk is $\mathrm{SL}(2, \mathrm{R})$. By using this property a localized state of a scalar field put inside the bulk of $\mathrm{AdS}_{3}$ space was constructed in [12] 14] 15] [13]. There have also been attempts to reconstruct bulk fields from the boundary CFT data by using bulk to boundary propagators. [16] [17] [18 In the case of 3d spin-3 gravity, however, we do not have embedding of the curved AdS spacetime including the internal space for $\mathrm{W}_{3}$ gauge symmetry within a higher-dimensional flat space. Best we can do is to make an assumption on the invariant subgroup according to symmetry considerations. At least for the localized state for a scalar field such an extension is straightforward.

\footnotetext{
${ }^{3}$ To switch to the other rule $\left(W_{n}\right)^{\dagger}=-W_{-n}$ we need to make a replacement $W_{n} \rightarrow i W_{n}$. This change can alternatively be performed by replacement of parameters $\alpha^{ \pm}, \beta^{ \pm}, \gamma \rightarrow i \alpha^{ \pm}, i \beta^{ \pm}, i \gamma$ in (4.1) in sec. 4.

${ }^{4}$ This space is associated with spin-3 gauge transformation. It is actually not an internal space; its coordinates mix with those of the real space under $\mathrm{W}_{3}$ transformations. However, in this paper we will call this space an 'internal space'.
} 
In the case of $\mathrm{AdS}_{3}$ gravity, where isometry is $\mathrm{SL}(2, \mathrm{R}) \otimes \mathrm{SL}(2, \mathrm{R})$, the condition for the localized state of a scalar field $|\psi\rangle$ put in the center of the bulk is given in terms of the generators of a sub-algebra $\operatorname{sl}(2, \mathrm{R})$ and given by [12] [14] 15$][13$

$$
\left(L_{n}-(-1)^{n} \bar{L}_{-n}\right)|\psi\rangle=0 \quad(n=-1,0,1)
$$

In the case of spin-3 gravity the symmetry algebra $\operatorname{sl}(3, \mathrm{R}) \oplus \operatorname{sl}(3, \mathrm{R})$ is generated by $L_{n}, \bar{L}_{n}(n=0, \pm 1)$ and $W_{n}, \bar{W}_{n}(n=0, \pm 1, \pm 2)$. For this theory conditions for a localized state of a scalar filed with $\mathrm{W}$ charge will be expressed in terms of generators of the $\mathrm{sl}(3, \mathrm{R})$ sub-algebra which contains the above $\mathrm{sl}(2, \mathrm{R})$ sub-algebra. Then the conditions for localized state will be (1.5) and 5

$$
\left(W_{n}-(-1)^{n} \bar{W}_{-n}\right)|\psi\rangle=0 \quad(n=-2,-1, \ldots, 2) .
$$

The first purpose of this paper is to construct a localized bulk state of a scalar field with a $\mathrm{W}_{3}$ charge in $3 \mathrm{~d}$ spin 3 gravity by solving (1.5) and (1.6) and study the equation of motion for the scalar field. We will work in Poincaré coordinates and denote $\operatorname{sl}(3, \mathrm{R})$ generators as $L_{n}^{h}$ and $W_{n}^{h}$. The generators without superscript $h, L_{n}$ and $W_{n}$, are generators in the elliptic basis for the global coordinates 13 . These are related by (2.13) and (2.18). To write down the equation of motion it is necessary to introduce coordinates $\alpha^{ \pm}, \beta^{ \pm}$and $\gamma$ for the 'internal space' 6 This higher-dimensional space is an analog of superspace in supersymmetric theory and this will be called W space in this paper. Structure of the W space obtained by combining 3d bulk spacetime and this 'internal space' will be elucidated. Metric of this W space (5.5) is shown to be a solution to higher-dimensional Einstein equation (5.6) with negative cosmological constant. The second purpose is to obtain an infinite dimensional representation in the bulk of the wedge mode algebra (1.4) in terms of differential operators $\partial_{x^{ \pm}}, \partial_{\alpha^{ \pm}}, \partial_{\beta^{ \pm}}, \partial_{\gamma}$ in addition to ordinary derivatives $\partial_{x^{ \pm}}, \partial_{y}$. Here $y$ is a radial coordinate. With this representation it is possible to obtain twoand three-point functions of quasi-primary operators $\Phi\left(x^{+}, \alpha^{+}, \beta^{+}, x^{-}, \alpha^{-}, \beta^{-}\right)$at $x^{ \pm}=t \pm x$ on the boundary and at $\left(\alpha^{ \pm}, \beta^{ \pm}\right)$in the 'internal space', which are actually descendants created from a Virasoro quasi-primary field $\Phi\left(x^{+}, x^{-}\right)$by $W_{-1}^{h}, W_{-2}^{h}$, $\bar{W}_{-1}^{h}$, and $\bar{W}_{-2}^{h}$. Similar type of correlation functions are computed by Fateev and Ribault in [20] by using their variables $x, y$, and $w$ for a representation of $\operatorname{sl}(3, \mathrm{R})$

\footnotetext{
${ }^{5}$ The localized state for a scalar field with distinct values of chiral and anti-chiral $\mathrm{W}$ charges $\mu$ and $\bar{\mu}$, the right hand side of the equation for $n=0$ needs to be replaced by a non-zero constant.

${ }^{6}$ These are sources for $W_{-2}^{h}, \bar{W}_{-2}^{h}, W_{-1}^{h}, \bar{W}_{-1}^{h}$, and $W_{0}^{h}+\bar{W}_{0}^{h}$. See (4.1).
} 
different from ours. Even if these variables may be related to our $x, \alpha$ and $\beta$ by suitable change of variables, the relation of the two sets of variables are not known. Hence it is necessary to construct the representation of $\mathrm{sl}(3, \mathrm{R})$ algebra in terms of $(x, \alpha, \beta)$ from scratch.

By using the above results it will be argued that the combined space of the bulk space and the internal space describes a renormalization group (RG) flow along $\beta^{ \pm}$coordinates as well as along holographic $y$ coordinate from an $\mathrm{AdS}_{3}$ vacuum with $W_{3}$ symmetry to another $\mathrm{AdS}_{3}$ vacuum. These two vacua are located on $3 \mathrm{D}$ hypersurfaces at $\beta^{ \pm}=0$ and $\beta^{ \pm}=\infty$, respectively. The AdS radii of the two $\mathrm{AdS}_{3}$ spaces are different by a factor 2. This flow is also confirmed by study of correlation functions. Two- and three-point functions of $\mathrm{W}$-descendants, which are quasi-primary fields moved in the internal space by $W_{-1}^{h}$ and $W_{-2}^{h}$, are computed 7 Two point function of quasi-primary chiral operators $\Phi_{h}\left(x^{+}, \alpha^{+}, \beta^{+}\right)$at $\alpha^{+}=\beta^{+}=$ 0 have the form

$$
\left\langle 0\left|\Phi_{h}\left(x_{1}^{+}, 0,0\right) \Phi_{h}\left(x_{2}^{+}, 0,0\right)\right| 0\right\rangle=\frac{1}{\left(x_{12}^{+}\right)^{2 h}} . \quad\left(x_{12}^{+} \equiv x_{1}^{+}-x_{2}^{+}\right)
$$

When the background for $\beta^{+}$is introduced, the two point function becomes complicated, (6.8), and when a limit $\beta^{+} \rightarrow \infty$ is taken, we have

$$
\lim _{\beta^{+} \rightarrow \infty}\left\langle 0\left|\left(\beta^{+}\right)^{h} \Phi_{h}\left(x_{1}^{+}, 0, \beta^{+}\right)\left(\beta^{+}\right)^{h} \Phi_{h}\left(x_{2}^{+}, 0, \beta^{+}\right)\right| 0\right\rangle=\frac{1}{\left(x_{12}^{+}\right)^{h}} .
$$

Hence the conformal weight changes as $h \rightarrow h / 2$.

Similar RG flow was reported in [22]8, where a solution to the equation of motion in $\operatorname{sl}(3, \mathrm{R}) \oplus \operatorname{sl}(3, \mathrm{R}) \mathrm{CS}$ theory of $3 \mathrm{~d}$ spin 3 gravity, which represents RG flow between $\mathrm{W}_{3}$ and $W_{3}^{(2)}\left[24\right.$ vacua, was obtained and it was shown that stress tensor $T_{U V}$ in the UV, which has conformal weights $(h, \bar{h})=(2,0)$, flows to an operator with $\left(h^{\prime}, \bar{h}^{\prime}\right)=(0,4)$ in the IR. Because the pattern of the change of the conformal weight is similar to our case, field theory is also expected to flow to $\mathrm{W}_{3}^{(2)}$ vacuum in the RG flow of the present paper. In sec.7 it is also shown that $L_{-1}^{h}$ in $\beta^{+}=0$ vacuum flows to $W_{-2}^{h}$ at $\beta^{+} \rightarrow \infty$. $W_{-2}^{h}$ is a 'new $L_{-1}^{h}$ ' for $W_{3}^{(2)}$ theory. However, properties of our flow and that in 22] are distinct: our flow is associated with a transformation of quasi-primary operators $\mathcal{O} \rightarrow e^{\beta^{+} W_{-1}^{h}} \mathcal{O} e^{-\beta^{+} W_{-1}^{h}}$. It will be argued in sec.7 that this flow is driven by the following additional terms in the action.

$$
\Delta S=\beta^{+}\left(-i W_{-1}^{h}\right)+\beta^{-}\left(-i \bar{W}_{-1}^{h}\right) .
$$

\footnotetext{
${ }^{7}$ Some properties of three-point functions of $\mathrm{W}$-descendats are discussed in 21 .

${ }^{8}$ Two vacua of spin-3 gravity is also discussed in [23].
} 
The generators $W_{-1}^{h}$ and $\bar{W}_{-1}^{h}$ are anti-hermitian, and their c-number counterparts are pure imaginary.

This paper is organized as follows. In sec.2 a boundary state in Poincaré coordinates is related to the primary state at the origin of Euclidean plane. In sec. 3 a localized state for a scalar field in the bulk is constructed for 3D spin 3 gravity. In sec. 4 an infinite dimensional representation of generators in the Poincaré coordinates, $L_{n}^{h}(n=-1,0,1)$ and $W_{n}^{h}(n=-2,-1,0,1,2)$, is constructed. In sec. 5 scalar equation of motion is derived and the metric tensor of the 8 dimensional space obtained by combining $3 \mathrm{~d}$ spacetime and the 'internal space' is obtained. This turns out a specific solution to the Einstein equation with negative cosmological constant. It is pointed out that this space describes a $R G$ flow from one $W_{3}$ vacuum to another. In sec. 6 two- and three-point functions of quasi-primary holomorphic operators $\Phi\left(x^{+}, \alpha^{+}, \beta^{+}\right)$on the boundary and the internal space are calculated by solving differential equations which comes from $\mathrm{W}_{3}$ symmetry of the vacuum. In sec. 7 it is shown that these correlation functions show a RG flow along the $\beta^{+}$ coordinate. It is also argued that this RG flow is triggered by adding extra terms to the action. Sec. 8 is left for summary. Two appendices A, B contain some technical details for obtaining costraints on the boundary state and the localized state in the bulk.

\section{A Boundary State}

In this section we will consider a localized state of a scalar field, which is extrapolated to the boundary. This state $|\psi\rangle_{B}$ is obtained from $|\psi\rangle$ by the following transformation [13].

$$
\begin{aligned}
|\psi\rangle_{B} & =\lim _{\rho \rightarrow \infty} g(\rho)|\psi\rangle, \\
g(\rho) & =e^{-\rho \frac{1}{2}\left(L_{1}-L_{-1}+\bar{L}_{1}-\bar{L}_{-1}\right)}
\end{aligned}
$$

The state on the boundary satisfies the following conditions in stead of (1.5) and (1.6).

$$
\begin{aligned}
& \lim _{\rho \rightarrow \infty} g(\rho)\left(L_{n}-(-1)^{n} \bar{L}_{-n}\right) g(\rho)^{-1}|\psi\rangle_{B}=0, \quad(n=-1,0,1), \\
& \lim _{\rho \rightarrow \infty} g(\rho)\left(W_{n}-(-1)^{n} \bar{W}_{-n}\right) g(\rho)^{-1}|\psi\rangle_{B}=0 \quad(n=-2,-1,0,1,2)
\end{aligned}
$$


Let us first consider (2.3). By working out several commutators the equations in (2.3) before the limit $\rho \rightarrow \infty$ are given by

$$
\begin{gathered}
{\left[\left(L_{0}-\bar{L}_{0}\right) \cosh \rho-\frac{1}{2}\left(L_{1}+L_{-1}-\bar{L}_{1}-\bar{L}_{-1}\right) \sinh \rho\right]|\psi\rangle_{B}=0} \\
{\left[\frac{1}{2}\left(L_{1}-L_{-1}+\bar{L}_{-1}-\bar{L}_{1}\right)+\frac{1}{2}\left(L_{1}+L_{-1}+\bar{L}_{-1}+\bar{L}_{1}\right) \cosh \rho\right.} \\
\left.-\left(L_{0}+\bar{L}_{0}\right) \sinh \rho\right]|\psi\rangle_{B}=0 \\
{\left[\frac{1}{2}\left(\bar{L}_{1}-\bar{L}_{-1}+L_{-1}-L_{1}\right)+\frac{1}{2}\left(L_{1}+L_{-1}+\bar{L}_{-1}+\bar{L}_{1}\right) \cosh \rho\right.} \\
\left.-\left(L_{0}+\bar{L}_{0}\right) \sinh \rho\right]|\psi\rangle_{B}=0 .
\end{gathered}
$$

In the limit $\rho \rightarrow \infty$ the above three conditions degenerate into two independent ones, and after taking appropriate linear combinations these are given by

$$
\begin{aligned}
& {\left[L_{0}-\frac{1}{2}\left(L_{1}+L_{-1}\right)\right]|\psi\rangle_{B}=0} \\
& {\left[\bar{L}_{0}-\frac{1}{2}\left(\bar{L}_{1}+\bar{L}_{-1}\right)\right]|\psi\rangle_{B}=0 .}
\end{aligned}
$$

A solution to these conditions are constructed on the primary state

$$
\left|O_{\Delta}\right\rangle=\lim _{t \rightarrow i \infty} O_{\Delta}(\phi=0, t)|0\rangle,
$$

where $O_{\Delta}(\phi, t)$ is a scalar primary operator with a conformal weight $(h, \bar{h})=$ $(\Delta / 2, \Delta / 2)$ and a scaling dimension $\Delta$ on the boundary. $\phi$ is an angular variable in the global coordinates. This state corresponds to a scalar operator put on the infinite past of the Euclidean boundary, and $|0\rangle$ is the vacuum in the global patch. This state satisfies 9

$$
\begin{array}{llrl}
L_{0}\left|O_{\Delta}\right\rangle & =\frac{\Delta}{2}\left|O_{\Delta}\right\rangle, & L_{1}\left|O_{\Delta}\right\rangle & =0 \\
\bar{L}_{0}\left|O_{\Delta}\right\rangle & =\frac{\Delta}{2}\left|O_{\Delta}\right\rangle, & \bar{L}_{1}\left|O_{\Delta}\right\rangle & =0 .
\end{array}
$$

It turns out the boundary state $|\psi\rangle_{B}$ is given by

$$
|\psi\rangle_{B}=e^{L_{-1}+\bar{L}_{-1}}\left|O_{\Delta}\right\rangle
$$

This is shown by directly checking (2.8)-(2.9). It can also be shown that the operator which acts on $|\psi\rangle_{B}$ in (2.8) agrees with the Virasoro generator $L_{1}^{h}$ in the hyperbolic

\footnotetext{
${ }^{9}$ For a while we will consider only the holomorphic and anti-holomorphic global Virasoro algebras.
} 
representation for the Poincaré coordinates. 13 .

$$
\begin{aligned}
L_{1}^{h} & =-L_{0}+\frac{1}{2}\left(L_{1}+L_{-1}\right), \\
L_{0}^{h} & =\frac{1}{2}\left(L_{1}-L_{-1}\right), \\
L_{-1}^{h} & =L_{0}+\frac{1}{2}\left(L_{1}+L_{-1}\right)
\end{aligned}
$$

There are similar relations for $\bar{L}_{n}^{h} . L_{0}^{h}$ is anti-hermitian and $L_{ \pm 1}^{h}$ are hermitian. The state $|\psi\rangle_{B}$ satisfies the quasi-primary conditions for Virasoro algebra in the Poincaré coordinates.

$$
\begin{aligned}
& L_{1}^{h}|\psi\rangle_{B}=\bar{L}_{1}^{h}|\psi\rangle_{B}=0, \\
& L_{0}^{h}|\psi\rangle_{B}=\frac{\Delta}{2}|\psi\rangle_{B}, \quad \bar{L}_{0}^{h}|\psi\rangle_{B}=\frac{\Delta}{2}|\psi\rangle_{B}
\end{aligned}
$$

It can be shown that the exponential operator which appears in (2.12) carries a quasi-primary state $\left|O_{\Delta}\right\rangle$ at the origin of Euclidean plane in the global coordinates to the point $(t, \phi)=(0,0)$ in the Lorentzian Poincaré coordinates.

Next we will consider the conditions (1.6) related to $\mathrm{W}$ generators. The transformed operators for finite $\rho$ are presented in appendix A. By sending the conditions (1.6) to the boundary, the following conditions for $|\psi\rangle_{B}$ are obtained in addition to (2.8) and (2.9).

$$
\begin{aligned}
& \left(W_{2}-4 W_{1}+6 W_{0}-4 W_{-1}+W_{-2}\right)|\psi\rangle_{B}=0 \\
& \left(\bar{W}_{2}-4 \bar{W}_{1}+6 \bar{W}_{0}-4 \bar{W}_{-1}+\bar{W}_{-2}\right)|\psi\rangle_{B}=0
\end{aligned}
$$

As in the case of the global Virasoro generators, these conditions define $W_{2}^{h}$ and $\bar{W}_{2}^{h}$ for the Poincaré coordinates in terms of those for global coordinates. Then the algebra (1.4) determines remaining $W_{n}^{h}$ 's.

$$
\begin{aligned}
W_{2}^{h} & =\frac{1}{4}\left(W_{2}-4 W_{1}+6 W_{0}-4 W_{-1}+W_{-2}\right) \\
W_{1}^{h} & =\frac{1}{4}\left(W_{2}-2 W_{1}+2 W_{-1}-W_{-2}\right) \\
W_{0}^{h} & =\frac{1}{4}\left(W_{2}-2 W_{0}+W_{-2}\right) \\
W_{-1}^{h} & =\frac{1}{4}\left(W_{2}+2 W_{1}-2 W_{-1}-W_{-2}\right) \\
W_{-2}^{h} & =\frac{1}{4}\left(W_{2}+4 W_{1}+6 W_{0}+4 W_{-1}+W_{-2}\right)
\end{aligned}
$$

Note that $W_{n}^{h}$ with even $n$ are hermitian operators and those with odd $n$ are antihermitian. A quasi-primary state of $\mathrm{W}_{3}$ algebra at the origin of Euclidean plane will 
be denoted as

$$
|\Delta, \mu\rangle=\lim _{t \rightarrow i \infty} O_{\Delta, \mu}(\phi=0, t)|0\rangle .
$$

This state satisfies the primary state conditions

$$
\begin{aligned}
& L_{1}|\Delta, \mu\rangle=0 \\
& L_{0}|\Delta, \mu\rangle=\frac{\Delta}{2}|\Delta, \mu\rangle, \\
& W_{2}|\Delta, \mu\rangle=W_{1}|\Delta, \mu\rangle=0, \\
& W_{0}|\Delta, \mu\rangle=\mu|\Delta, \mu\rangle
\end{aligned}
$$

and similar conditions for $\bar{W}_{n}$. The boundary state

$$
\left|\psi_{\Delta, \mu}\right\rangle_{B}=e^{L_{-1}+\bar{L}_{-1}}|\Delta, \mu\rangle
$$

satisfies (2.14)-(2.15) and

$$
\begin{aligned}
& W_{2}^{h}\left|\psi_{\Delta, \mu}\right\rangle_{B}=W_{1}^{h}\left|\psi_{\Delta, \mu}\right\rangle_{B}=0, \\
& W_{0}^{h}\left|\psi_{\Delta, \mu}\right\rangle_{B}=\mu\left|\psi_{\Delta, \mu}\right\rangle_{B}
\end{aligned}
$$

and similar conditions with respect to $\bar{W}_{n}^{h}$.

\section{Construction of A Localized State}

We will solve the conditions (1.5) and (1.6) for the localized state $|\psi\rangle$. This state will be built on the boundary state $|\psi\rangle_{B}$.

First we will consider the case of $\mathrm{AdS}_{3}$ and solve (1.5) without $\mathrm{W}$ extension. Although this state was solved in [12] by a series expansion, we will obtain the state here in terms of an integral representation. The localized state is represented in a form

$$
|\psi\rangle=\int d x d \bar{x} f(x, \bar{x}) e^{x L_{-1}^{h}+\bar{x} \bar{L}_{-1}^{h}}\left|\psi_{\Delta}\right\rangle_{B}
$$

Let us first consider the condition (1.5) with $n=0$. When $L_{0}$ is applied on $|\psi\rangle$, we have

$$
\begin{aligned}
L_{0}^{h}|\psi\rangle & =\int d x d \bar{x} f(x, \bar{x}) L_{0}^{h} e^{x L_{-1}^{h}+\bar{x} \bar{L}_{-1}^{h}}\left|\psi_{\Delta}\right\rangle_{B} \\
& =\int d x d \bar{x} f(x, \bar{x}) e^{x L_{-1}^{h}}\left(e^{-x L_{-1}^{h}} L_{0}^{h} e^{x L_{-1}^{h}}\right) e^{\bar{x} \bar{L}_{-1}^{h}}\left|\psi_{\Delta}\right\rangle_{B}
\end{aligned}
$$

We work out the commutator $e^{-x L_{-1}^{h}} L_{0}^{h} e^{x L_{-1}^{h}}=L_{0}^{h}+x L_{-1}^{h}$ and replace $L_{0}^{h}$ by $\Delta / 2$ according to (2.15), and $L_{-1}^{h}$ by $\partial_{x}$ due to exponential $e^{x L_{-1}^{h}}$ in the integrand. It is 
assumed that the surface term drops out. Finally by performing partial integration, we have

$$
L_{0}^{h}|\psi\rangle=\int d x d \bar{x}\left(\frac{\Delta}{2} f(x, \bar{x})-x \partial_{x} f(x, \bar{x})\right) e^{x L_{-1}^{h}+\bar{x} \bar{L}_{-1}^{h}}\left|\psi_{\Delta}\right\rangle_{B}
$$

By a similar rewriting of $\bar{L}_{0}^{h}|\psi\rangle$, the condition $\left(L_{0}^{h}-\bar{L}_{0}^{h}\right)|\psi\rangle=0$ yields an equation.

$$
\left(x \partial_{x}-\bar{x} \partial_{\bar{x}}\right) f(x, \bar{x})=0
$$

The other two conditions in (1.5) produce the following equations.

$$
\begin{aligned}
& \left(x^{2} \partial_{x}+\Delta x\right) f(x, \bar{x})=0, \\
& \left(\bar{x}^{2} \partial_{\bar{x}}+\Delta \bar{x}\right) f(x, \bar{x})=0
\end{aligned}
$$

Solution to the above equations is given up to a multiplicative constant by $f(x, \bar{x})=$ $(1+x \bar{x})^{\Delta-2}$, and the localized state is obtained.

$$
|\psi\rangle=\int d x d \bar{x}(1+x \bar{x})^{\Delta-2} e^{x L_{-1}^{h}+\bar{x} \bar{L}_{-1}^{h}}\left|\psi_{\Delta}\right\rangle_{B}
$$

To carry out the integration, we put $x=r e^{i \theta}$ and $\bar{x}=r e^{-i \theta}$. After expanding the exponential in powers of $r$ we carry out the $\theta$ integral. Then $r$ integral is performed term by term via analytical continuation in $\Delta$, assuming that the sum and $r$ integration can be exchanged.

$$
|\psi\rangle=\frac{\pi}{1-\Delta} \sum_{n=0}^{\infty} \frac{(-1)^{n}}{n !(\Delta)_{n}}\left(L_{-1}^{h} \bar{L}_{-1}^{h}\right)^{n}\left|\psi_{\Delta}\right\rangle_{B}
$$

Here $(\Delta)_{n}=\Delta(\Delta+1) \ldots(\Delta+n-1)$. This expression of the localized state coincides with that obtained by a different method in [12]. Equivalence with the HKLL construction is shown in Appendix A of [13].

In the case of spin 3 gravity, the localized state is obtained in a similar way. It is obtained as an integral in a form

$$
\begin{aligned}
& |\psi\rangle=\int d x d \bar{x} d y d \bar{y} d z d \bar{z} F(x, y, z, \bar{x}, \bar{y}, \bar{z}) e^{x W_{-2}^{h}} e^{y W_{-1}^{h}} e^{z L_{-1}^{h}} \\
& e^{\bar{x} \bar{W}_{-2}^{h}} e^{\bar{y} \bar{W}_{-1}^{h}} e^{\bar{z} \bar{L}_{-1}^{h}}\left|\psi_{\Delta, \mu}\right\rangle_{B} .
\end{aligned}
$$

Here $\bar{x}, \bar{y}, \bar{z}$ are complex conjugates of $x, y, z$, respectively. These variables have nothing to do with the coordinates of spacetime. They are used only in this section and appendix B. The function $F$ is obtained by the same method as above for $\mathrm{AdS}_{3}$ gravity and the details are given in appendix $\mathrm{B}$. The result is

$$
F(x, y, z, \bar{x}, \bar{y}, \bar{z})=\left[\frac{R_{+}}{R_{-}}\right]^{\frac{3}{4} \mu} T^{\frac{\Delta-8}{4}}
$$


where

$$
\begin{aligned}
R_{+}= & 1+2 \zeta\left|1+\xi_{2}\right|^{2}+\zeta^{2}\left|1-4 \xi_{1}-2 \xi_{2}-\xi_{2}^{2}\right|^{2}, \\
R_{-}= & 1+2 \zeta\left|1-\xi_{2}\right|^{2}+\zeta^{2}\left|1+4 \xi_{1}+2 \xi_{2}-\xi_{2}^{2}\right|^{2}, \\
T= & 1+4 \zeta\left(1+\left|\xi_{2}\right|^{2}\right)+2 \zeta^{2}\left\{\left|4 \xi_{1}+2 \bar{\xi}_{2}\right|^{2}+3\left|\xi_{2}^{2}-1\right|^{2}\right\} \\
& +4 \zeta^{3}\left(1+\left|\xi_{2}\right|^{2}\right)^{-1}\left\{\left.|1+| \xi_{2}\right|^{4}-\xi_{2}^{2}-\left.\bar{\xi}_{2}^{2}\right|^{2}\right. \\
& \left.+\left|4\left(1+\left|\xi_{2}\right|^{2}\right) \xi_{1}+3 \xi_{2}-\xi_{2}^{3}+\bar{\xi}_{2}+\xi_{2}^{2} \bar{\xi}_{2}\right|^{2}\right\} \\
& +\zeta^{4}\left|1-16 \xi_{1}^{2}-16 \xi_{1} \xi_{2}-6 \xi_{2}^{2} \xi_{2}^{4}\right|^{2} .
\end{aligned}
$$

Here $\zeta, \xi_{i}$ are variables defined by

$$
\zeta=z \bar{z}=|z|^{2}, \quad \xi_{1}=\frac{x}{z^{2}}, \quad \xi_{2}=\frac{y}{z}, \quad \bar{\xi}_{1}=\frac{\bar{x}}{\bar{z}^{2}}, \quad \bar{\xi}_{2}=\frac{\bar{y}}{\bar{z}} .
$$

Hence an integral representation of the localized state is obtained. $R_{+}, R_{-}$and $T$ are manifestly positive definite. The integral in (3.9) may be carried out by analytical continuation with respect to $\Delta$, and the solution to the condition (1.6) will exist. The integral is not yet evaluated explicitly. Finally, notice that no relation between $\Delta$ and $\mu$ is required for existence of the localized state.

\section{Infinite Dimensional Representation of $L_{n}^{h}$ and $W_{n}^{h}$ in the Bulk}

The localized state obtained in the previous section can be moved to arbitrary positions at $\left(x^{+}, x^{-}, y\right)$ in the bulk by unitary transformations. It is also possible to carry out extra $\mathrm{W}_{3}$ transformations on this state. This will move the state in the 'internal space'. In the following we will work out this construction in Poincaré coordinates. As in [13] the explicit form of the localized state in the product of the bulk space and 'internal space' will be given by

$$
\begin{aligned}
& \left|\Phi\left(x^{+}, \alpha^{+}, \beta^{+}, x^{-}, \alpha^{-}, \beta^{-}, y, \gamma\right)\right\rangle \\
= & e^{i x^{+} L_{-1}^{h}} e^{i x^{-} \bar{L}_{-1}^{h}} e^{i \alpha^{+} W_{-2}^{h}} e^{i \alpha^{-} \bar{W}_{-2}^{h}} e^{\beta^{+} W_{-1}^{h}} e^{\beta^{-} \bar{W}_{-1}^{h}} e^{-\frac{i}{2} \gamma\left(W_{0}^{h}+\bar{W}_{0}^{h}\right)} y^{L_{0}^{h}+\bar{L}_{0}^{h}}|\phi\rangle,
\end{aligned}
$$

where $x^{ \pm}=t \pm x$ are light-cone coordinates on the boundary in Poincaré coordinates, $y$ is the radial coordinate, and $\alpha^{ \pm}$and $\beta^{ \pm}, \gamma$ are parameters of the global $\mathrm{W}_{3}$ transformation in the $c \rightarrow \infty$ limit.

By studying $S L(3, R) \otimes S L(3, R)$ transformation of this localized state at arbitrary point we will be able to obtain representation of $L_{n}^{h}$ and $W_{n}^{h}$ in the bulk in 
terms of differential operators. This is the purpose of this section. First, let us apply $e^{-\epsilon L_{-1}^{h}}$ on $|\Phi\rangle$, where $\epsilon$ is an infinitesimal parameter. We have

$$
\begin{aligned}
& e^{-\epsilon L_{-1}^{h}}\left|\Phi\left(x^{+}, \alpha^{+}, \beta^{+}, x^{-}, \alpha^{-}, \beta^{-}, y, \gamma\right)\right\rangle=\left|\Phi\left(x^{+}+i \epsilon, \alpha^{+}, \beta^{+}, x^{-}, \alpha^{-}, \beta^{-}, y, \gamma\right)\right\rangle \\
& \equiv e^{\epsilon \hat{L}_{-1}^{h}}\left|\Phi\left(x^{+}, \alpha^{+}, \beta^{+}, x^{-}, \alpha^{-}, \beta^{-}, y, \gamma\right)\right\rangle
\end{aligned}
$$

and this defines a differential operator $\hat{L}_{-1}^{h}=i \partial_{x^{+}} 10$ For the other generators the exponential of the generator is moved to the right by using the $\mathrm{W}_{3}$ algebra until it reaches the localized operator $|\phi\rangle$ at the central point, and the conditions (1.5) and (1.6) are used and then the exponential is moved back to the left. By this procedure we identify the following representation.

$$
\begin{aligned}
\hat{L}_{-1}^{h}= & i \partial_{+}, \\
\hat{L}_{0}^{h}= & -x^{+} \partial_{+}-2 \alpha^{+} \partial_{\alpha^{+}}-\beta^{+} \partial_{\beta^{+}}-\frac{1}{2} y \partial_{y}, \\
\hat{L}_{1}^{h}= & -i\left[\left(x^{+}\right)^{2}-3\left(\beta^{+}\right)^{2}\right] \partial_{+}-i x^{+} y \partial_{y}-3 i \beta^{+} \partial_{\gamma}+i\left[2\left(\beta^{+}\right)^{3}-4 x^{+} \alpha^{+}\right] \partial_{\alpha^{+}} \\
& -i\left[2 x^{+} \beta^{+}+4 \alpha^{+}\right] \partial_{\beta^{+}}-i y^{2} \cos (2 \gamma) \partial_{-}-i y^{2} \cos (2 \gamma) \beta^{-} \partial_{\alpha^{-}}-i y^{2} \sin (2 \gamma) \partial_{\beta^{-}},
\end{aligned}
$$

$$
\begin{aligned}
\hat{W}_{-2}^{h}= & i \partial_{\alpha^{+}} \\
\hat{W}_{-1}^{h}= & -x^{+} \partial_{\alpha^{+}}-\partial_{\beta^{+}}, \\
\hat{W}_{0}^{h}= & 2 i \beta^{+} \partial_{+}+i\left[-\left(x^{+}\right)^{2}+\left(\beta^{+}\right)^{2}\right] \partial_{\alpha^{+}}-2 i x^{+} \partial_{\beta^{+}}-i \partial_{\gamma}, \\
\hat{W}_{1}^{h}= & 3 x^{+} \partial_{\gamma}+\left[4 \alpha^{+}-6 x^{+} \beta^{+}\right] \partial_{+}+\left[\left(x^{+}\right)^{3}-3 x^{+}\left(\beta^{+}\right)^{2}\right] \partial_{\alpha^{+}}+\left[3\left(x^{+}\right)^{2}-\left(\beta^{+}\right)^{2}\right] \partial_{\beta^{+}} \\
& -\beta^{+} y \partial_{y}-y^{2} \sin (2 \gamma) \partial_{-}-\beta^{-} y^{2} \sin (2 \gamma) \partial_{\alpha^{-}}+y^{2} \cos (2 \gamma) \partial_{\beta^{-}}, \\
\hat{W}_{2}^{h}= & -i\left[3\left(\beta^{+}\right)^{4}-\left(x^{+}\right)^{4}-16\left(\alpha^{+}\right)^{2}+6\left(x^{+}\right)^{2}\left(\beta^{+}\right)^{2}\right] \partial_{\alpha^{+}} \\
& +i\left[y^{4}+4 y^{2} \beta^{-} \beta^{+} \cos (2 \gamma)-4 y^{2} \beta^{-} x^{+} \sin (2 \gamma)\right] \partial_{\alpha^{-}} \\
& +i\left[16 \alpha^{+} \beta^{+}-4 x^{+}\left(\beta^{+}\right)^{2}+4\left(x^{+}\right)^{3}\right] \partial_{\beta^{+}} \\
& +i y^{2}\left[4 \beta^{+} \sin (2 \gamma)+4 x^{+} \cos (2 \gamma)\right] \partial_{\beta^{-}}-i\left[4\left(\beta^{+}\right)^{3}+12 \beta^{+}\left(x^{+}\right)^{2}-16 x^{+} \alpha^{+}\right] \partial_{+} \\
& +i y^{2}\left[4 \beta^{+} \cos (2 \gamma)-4 x^{+} \sin (2 \gamma)\right] \partial_{-}+i\left[6\left(\beta^{+}\right)^{2}+6\left(x^{+}\right)^{2}\right] \partial_{\gamma} \\
& +i\left[8 \alpha^{+}-4 x^{+} \beta^{+}\right] y \partial_{y}
\end{aligned}
$$

Here $\partial_{ \pm}$denotes $\partial_{x^{ \pm}}$for simplicity. The expressions for $\hat{\bar{L}}_{n}^{h}$ and $\hat{\bar{W}}_{n}^{h}$ are obtained by an exchange $+\leftrightarrow-$. These generators satisfy the wedge-mode algebra (1.4). In the remaining part of this paper, the 'hat' for the differential operators will be omitted for simplicity of notation.

\footnotetext{
${ }^{10}$ The flip of sign in front of $\epsilon$ is necessary to insure correct algebra.
} 


\section{Scalar Equation of Motion and W Geometry as RG Flow}

The localized state obtained in the preceding section satisfy a differential equation, which also depends on differential operators $\partial_{\alpha^{ \pm}}, \partial_{\beta^{ \pm}}$, and $\partial_{\gamma}$. This is derived by using quadratic Casimir operator of $\operatorname{SL}(3, \mathrm{R})$ like $\mathrm{SL}(2, \mathrm{R})$ case 15] [13]. This is given by

$$
\begin{aligned}
C_{2}(L, W)= & \left(L_{0}^{h}\right)^{2}-\frac{1}{2}\left(L_{1}^{h} L_{-1}^{h}+L_{-1}^{h} L_{1}^{h}\right)+\frac{1}{8}\left(W_{2}^{h} W_{-2}^{h}+W_{-2}^{h} W_{2}^{h}\right) \\
& -\frac{1}{2}\left(W_{1}^{h} W_{-1}^{h}+W_{-1}^{h} W_{1}^{h}\right)+\frac{3}{4}\left(W_{0}^{h}\right)^{2} .
\end{aligned}
$$

By using

$$
\left(C_{2}(L, W)+C_{2}(\bar{L}, \bar{W})\right)\left|O_{\Delta, \mu}\right\rangle=\frac{1}{2}\left\{\Delta^{2}-8 \Delta+3 \mu^{2}\right\}\left|O_{\Delta, \mu}\right\rangle,
$$

and the representation (4.4), Klein-Gordon equation in W space is derived.

$$
\begin{aligned}
& {\left[y^{2} \partial_{y}^{2}-7 y \partial_{y}-3 \partial_{\gamma}^{2}-4 y^{2} \cos 2 \gamma \partial_{x^{+}} \partial_{x^{-}}-4 y^{2} \cos 2 \gamma\left(\beta^{-} \partial_{x^{+}} \partial_{\alpha^{-}}+\beta^{+} \partial_{x^{-}} \partial_{\alpha^{+}}\right)\right.} \\
& -4 y^{2} \sin 2 \gamma\left(\partial_{x^{+}} \partial_{\beta^{-}}+\partial_{x^{-}} \partial_{\beta^{+}}\right)-4 y^{2} \sin 2 \gamma\left(\beta^{+} \partial_{\alpha^{+}} \partial_{\beta^{-}}+\beta^{-} \partial_{\alpha^{-}} \partial_{\beta^{+}}\right) \\
& \left.-\left(y^{4}+4 \beta^{+} \beta^{-} y^{2} \cos 2 \gamma\right) \partial_{\alpha^{+}} \partial_{\alpha^{-}}+4 y^{2} \cos 2 \gamma \partial_{\beta^{+}} \partial_{\beta^{-}}-m^{2} \ell_{A d S}^{2}\right]|\Phi\rangle=0,
\end{aligned}
$$

where $m$ is a mass of the scalar field, $\ell_{A d S}$ AdS length, and $m^{2} \ell_{A d S}^{2}=\Delta^{2}-8 \Delta+3 \mu^{2}$, which gives

$$
\Delta=4+\sqrt{m^{2} \ell_{A d S}^{2}+16-3 \mu^{2}} .
$$

$\mathrm{Eq}(5.3)$ is an equation of motion for a scalar field propagating in an $8 \mathrm{~d}$ spacetime with the metric field given by

$$
\begin{aligned}
d s_{8}^{2}= & { }^{(8)} g_{M N} d x^{M} d x^{N} \\
= & y^{-2} d y^{2}-y^{-4}\left(y^{2} \cos 2 \gamma+4 \beta^{+} \beta^{-}\right) d x^{+} d x^{-}-4 y^{-4} d \alpha^{+} d \alpha^{-} \\
& +y^{-2} \cos 2 \gamma d \beta^{+} d \beta^{-}+4 y^{-4}\left(\beta^{+} d x^{+} d \alpha^{-}+\beta^{-} d x^{-} d \alpha^{+}\right) \\
& -y^{-2} \sin 2 \gamma\left(d x^{+} d \beta^{-}+d x^{-} d \beta^{+}\right)-\frac{1}{3} d \gamma^{2},
\end{aligned}
$$

where $x^{M}=\left(x^{+}, x^{-}, y, \alpha^{+}, \alpha^{-}, \beta^{+}, \beta^{-}, \gamma\right)$. The determinant of this metric is ${ }^{(8)} g=$ $\operatorname{det}{ }^{(8)} g_{M N}=\frac{1}{12} y^{-18}$. It can be shown that this metric satisfies Einstein equation,

$$
{ }^{(8)} R_{M N}-\frac{1}{2}{ }^{(8)} g_{M N}{ }^{(8)} R=-\Lambda_{8}{ }^{(8)} g_{M N}
$$

with a negative cosmological constant $\Lambda_{8}=-36$, where the cosmological constant of the original $\mathrm{AdS}_{3}$ is $\Lambda_{3}=-1$ ( $\ell_{\mathrm{AdS}}=1$ in our units). The metric (5.5) is, however, 
not the one of $\mathrm{AdS}_{8}$ space. It has signature $(+,+,+,+,-,-,-,-)$ and isometry of (5.5) is only $\mathrm{SL}(3, \mathrm{R}) \otimes \mathrm{SL}(3, \mathrm{R})$. This is an analog of superspace in supersymmetric theory, because commutators of $W_{m}$ 's are given in terms of $L_{n}$ (1.4).

The original physical $\mathrm{AdS}_{3}$ space is the hypersurface embedded at $\alpha^{ \pm}=\beta^{ \pm}=$ $\gamma=0$ in this $\mathrm{W}$ space. Other hypersurfaces with constant non-vanishing $\alpha^{ \pm}, \beta^{ \pm}$ and $\gamma$ may also be considered. An induced metric on this hypersurface is given by

$$
\left.d s_{8}^{2}\right|_{\text {hypersurface }}=y^{-2} d y^{2}-y^{-4}\left(y^{2} \cos 2 \gamma+4 \beta^{+} \beta^{-}\right) d x^{+} d x^{-} .
$$

Property of the hypersurface depends on the values of $\gamma$ and $\beta^{ \pm}$.

- When $\beta^{+}, \beta^{-}=0$ and $-\frac{\pi}{4}<\gamma<\frac{\pi}{4}$, the hypersurface corresponds to an ordinary $\mathrm{AdS}_{3}$ vacuum with $\mathrm{AdS}$ length $\ell_{\mathrm{A} d S}$.

- When $\beta^{+} \beta^{-}>0$ and $\gamma= \pm \frac{\pi}{4}, \beta^{ \pm}$can be absorbed by rescaling of $y$ and the hypersurface is again $\mathrm{AdS}_{3}$ but with other value of AdS length $\ell_{\text {AdS }}^{\prime}=$ $\frac{1}{2} \ell_{\text {AdS }}=\frac{1}{2}$, because $y^{-2} d y^{2}=\frac{1}{4}\left(y^{2}\right)^{-2}\left(d\left(y^{2}\right)\right)^{2}$.

- For $\gamma \neq \pm \frac{\pi}{4}$ and $\beta^{+} \beta^{-}>0$ the spacetime on the hypersurface is not AdS, but asymptotically AdS. This is a solution interpolating two vacua: one corresponding to UV CFT at $y=0$ with a AdS length $\ell_{\mathrm{AdS}}^{\prime}$, and the other to IR CFT at $y=\infty$ with $\ell_{\text {AdS }}$. Hence conformal symmetry is broken in the boundary field theory for non-zero $\beta^{+} \beta^{-}$. When $\beta^{ \pm} \rightarrow \infty$, conformal symmetry is recovered.

This observation suggests that the parameters $\beta^{ \pm}$also play the role of holographic renormalization group scales, which the radial variable $y$ plays in the conventional AdS/CFT correspondence 11 when the values of $\beta^{ \pm}$are sent to infinity, then the dual CFT may flow to another one. 12 Metric (5.5) appears to describe a structure of renormalization group flows between $\mathrm{W}_{3}$ vacua. It is stressed that the physical spacetime is a 3D hypersurface with constant $\alpha^{ \pm}, \beta^{ \pm}$and $\gamma$ embedded in the $8 \mathrm{D}$ space. As the values of these variables are changed, the spacetime as well as the dual CFT change just like they do as the radial variable $y$ changes.

\footnotetext{
${ }^{11}$ Restricting the bulk fields to a hypersurface with fixed values of $\alpha, \beta, \gamma$ is equivalent to transforming all the fields by $e^{i \alpha W_{-2}^{h}} e^{\beta W_{-1}^{h}} e^{\frac{i}{2} \gamma W_{0}^{h}}$ with these values as fixed backgrounds. This transformation may be equivalent to adding some perturbations to the action integral.

${ }^{12}$ In 22 a solution to equation of motion for $\mathrm{sl}(3, \mathrm{R}) \otimes \mathrm{sl}(3, \mathrm{R}) \mathrm{CS}$ gauge theory which interpolates between two vacua was presented. This solution agrees with the induced metric (5.7). This fact may be an evidence that (3.9) is a correct localized state in 3D spin-3 gravity.
} 
This view point is interesting and worth further investigation. In sec. 6 twoand three-point correlation functions of quasi-primary operators in $\mathrm{W}_{3}$-extended CFT are calculated, and in sec. 7 it is shown that introduction of infinite-valued $\beta^{ \pm}$background to the quasi-primaries actually modifies the conformal weights of quasi-primary fields.

\section{Correlation Functions on the Boundary}

In the preceding section representation (4.4) of W-algebra generators in the bulk in terms of differential operators are obtained. In this section this result will be used to to study correlation functions of quasi-primary operators. In the case of global Virasoro algebra the descendants of quasi-primary states $\left|O_{\Delta}\right\rangle$ are given by $\left(L_{-1}\right)^{n}\left|O_{\Delta}\right\rangle(n=1,2, \ldots)$ and these states are put into one to one correspondence with local states at arbitrary boundary points by a map $\left|\psi\left(x^{+}\right)\right\rangle=e^{i x^{+} L_{-1}}\left|O_{\Delta}\right\rangle$. Actually, this new state is a quasi-primary state with respect to new generators $L_{1}\left(x^{+}\right)$and $L_{0}\left(x^{+}\right)$at point $x^{+}$. Hence descendants created by the raising operators of the wedge algebra plays an important role in the representation theory. In the case of $\mathrm{W}_{3}$ algebra (in the $c \rightarrow \infty$ limit), $W_{-2}$ and $W_{-1}$ are such raising operators and it will be natural to introduce an 'internal space' with coordinates $\alpha^{ \pm}$and $\beta^{ \pm}$in accord with (4.1). Descendants generated by $W_{-2}$ and $W_{-1}$ are organized into quasiprimary states with respect to new generators $L_{n}\left(x^{+}, \alpha^{+}, \beta^{+}\right)$and $W_{n}\left(x^{+}, \alpha^{+}, \beta^{+}\right)$ at point $\left(\alpha^{+}, \beta^{+}\right)$in the 'internal space', as well as point $x^{+}$on the boundary. Because the commutators of $W_{n}$ 's are $L_{m}$, the coordinates of this 'internal space' mix with those of the real $2 \mathrm{~d}$ spacetime, $x^{+}$, under $\mathrm{SL}(3, \mathrm{R})$ transformation.

We need representation of the holomorphic $\mathrm{SL}(3, \mathrm{R})$ generators on the boundary. This is done by replacement

$$
\begin{aligned}
& y \partial_{y} \rightarrow \Delta=2 h \\
& \partial_{\gamma} \rightarrow-i \mu .
\end{aligned}
$$

and a subsequent limit $y \rightarrow 0$ in (4.4).

$$
\begin{aligned}
L_{-1}^{h}= & i \partial_{+}, \\
L_{0}^{h}= & -x^{+} \partial_{+}-2 \alpha^{+} \partial_{\alpha^{+}}-\beta^{+} \partial_{\beta^{+}}-\frac{1}{2} \Delta, \\
L_{1}^{h}= & -i\left[\left(x^{+}\right)^{2}-3\left(\beta^{+}\right)^{2}\right] \partial_{+}-3 \mu \beta^{+}+i\left[2\left(\beta^{+}\right)^{3}-4 x^{+} \alpha^{+}\right] \partial_{\alpha^{+}} \\
& -i\left[2 x^{+} \beta^{+}+4 \alpha^{+}\right] \partial_{\beta^{+}}-i \Delta x^{+}
\end{aligned}
$$




$$
\begin{aligned}
W_{-2}^{h}= & i \partial_{\alpha^{+}}, \\
W_{-1}^{h}= & -x^{+} \partial_{\alpha^{+}}-\partial_{\beta^{+}}, \\
W_{0}^{h}= & 2 i \beta^{+} \partial_{+}+i\left[-\left(x^{+}\right)^{2}+\left(\beta^{+}\right)^{2}\right] \partial_{\alpha^{+}}-2 i x^{+} \partial_{\beta^{+}}-\mu, \\
W_{1}^{h}= & {\left[4 \alpha^{+}-6 x^{+} \beta^{+}\right] \partial_{+}+\left[\left(x^{+}\right)^{3}-3 x^{+}\left(\beta^{+}\right)^{2}\right] \partial_{\alpha^{+}} } \\
& +\left[3\left(x^{+}\right)^{2}-\left(\beta^{+}\right)^{2}\right] \partial_{\beta^{+}}-\Delta \beta^{+}-3 i \mu x^{+}, \\
W_{2}^{h}= & -i\left[3\left(\beta^{+}\right)^{4}-\left(x^{+}\right)^{4}-16\left(\alpha^{+}\right)^{2}+6\left(x^{+}\right)^{2}\left(\beta^{+}\right)^{2}\right] \partial_{\alpha^{+}} \\
& +i\left[16 \alpha^{+} \beta^{+}-4 x^{+}\left(\beta^{+}\right)^{2}+4\left(x^{+}\right)^{3}\right] \partial_{\beta^{+}} \\
& -i\left[4\left(\beta^{+}\right)^{3}+12 \beta^{+}\left(x^{+}\right)^{2}-16 x^{+} \alpha^{+}\right] \partial_{+} \\
& +6 \mu\left[\left(\beta^{+}\right)^{2}+\left(x^{+}\right)^{2}\right]+i \Delta\left[8 \alpha^{+}-4 x^{+} \beta^{+}\right]
\end{aligned}
$$

These generators satisfy $\mathrm{sl}(3, \mathrm{R})$ algebra (1.4). Similar representation is presented in sec 15.7.4 of [19]. 13 This representation of $\mathrm{sl}(3, \mathrm{R})$, however, differs from (6.4). Although this representation may be made coincide with (6.4) by change of variables, in order to find out appropriate way of changing variables, if any, knowledge of (6.4) itself is necessary. Because variables $\alpha^{+}$and $\beta^{+}$correspond to $W_{-2}^{h}$ and $W_{-1}^{h}$ descendants, (6.4) must be used for our purpose.

Let $O_{\Delta_{i}, \mu_{i}}\left(x_{i}^{+}, \alpha_{i}^{+}, \beta_{i}^{+}\right)(i=1,2)$ be two holomorphic quasi-primary operators at points $\left(x_{i}^{+}, \alpha_{i}^{+}, \beta_{i}^{+}\right)$in the $\mathrm{W}$ space. Due to invariance of the vacuum two-point function of these operators,

$$
G_{12}\left(x_{1}^{+}, \alpha_{1}^{+}, \beta_{1}^{+} ; x_{2}^{+}, \alpha_{2}^{+}, \beta_{2}^{+}\right)=\left\langle 0\left|T O_{\Delta_{1}, \mu_{1}}\left(x_{1}^{+}, \alpha_{1}^{+}, \beta_{1}^{+}\right) O_{\Delta_{2}, \mu_{2}}\left(x_{2}^{+}, \alpha_{2}^{+}, \beta_{2}^{+}\right)\right| 0\right\rangle
$$

should satisfy the following differential equations. $T$ is the time-ordering prescription 14

$$
\begin{gathered}
\left(L_{n}^{h(1)}+L_{n}^{h(2)}\right) G_{12}=0 \\
\left(W_{n}^{h(1)}+W_{n}^{h(2)}\right) G_{12}=0
\end{gathered}
$$

Here superscripts (1) and (2) on $L_{n}^{h}$ and $W_{n}^{h}$ refer to each operator $O_{\Delta_{i}, \mu_{i}}$. This two-point function is non-vanishing, if and only if $\Delta_{1}=\Delta_{2}$ and $\mu_{1}+\mu_{2}=0$ are satisfied. Condition stemming from $L_{-1}^{h}$ restricts $G_{12}$ to depend on $x_{1}^{+}$and $x_{2}^{+}$only through a combination $x_{12}^{+}=x_{1}^{+}-x_{2}^{+}$. Similarly, due to the condition from $W_{-2}^{h}$,

\footnotetext{
${ }^{13}$ See also eqs $(2.25)-(2.32)$ of $[20]$.

${ }^{14}$ Correlation functions in $\mathrm{W}_{3}$ CFT are computed by Fateev and Ribault in [20] by using the same methods and by using representation of $\operatorname{sl}(3, \mathrm{R})$ in terms of their variables $(x, y, w)$. Connection of these variables to our $\left(x^{+}, \alpha^{+}, \beta^{+}\right)$is not known.
} 
$G_{12}$ is a function of $\alpha_{12}^{+}=\alpha_{1}^{+}-\alpha_{2}^{+}$. The remaining equations in (6.6) and (6.7) determine $G_{12}$ up to an overall constant. For a time order $t_{1}>t_{2}$ it is given by

$$
G_{12}=\left(D_{12}\right)^{-\frac{1}{4} \Delta_{1}+\frac{3}{4} \mu_{1}}\left(D_{12}^{*}\right)^{-\frac{1}{4} \Delta_{1}-\frac{3}{4} \mu_{1}}
$$

Here $D_{12}$ and $D_{12}^{*}$ are defined by

$$
\begin{aligned}
& D_{12}=\left(x_{1}^{+}-x_{2}^{+}\right)^{2}+\left(\beta_{1}^{+}-\beta_{2}^{+}\right)^{2}+2 i\left(x_{1}^{+}-x_{2}^{+}\right)\left(\beta_{1}^{+}+\beta_{2}^{+}\right)-4 i\left(\alpha_{1}^{+}-\alpha_{2}^{+}\right), \\
& D_{12}^{*}=\left(x_{1}^{+}-x_{2}^{+}\right)^{2}+\left(\beta_{1}^{+}-\beta_{2}^{+}\right)^{2}-2 i\left(x_{1}^{+}-x_{2}^{+}\right)\left(\beta_{1}^{+}+\beta_{2}^{+}\right)+4 i\left(\alpha_{1}^{+}-\alpha_{2}^{+}\right) .
\end{aligned}
$$

Two-point function for other time ordering is obtained by $t \rightarrow t-i \epsilon$ prescription. For completeness transformation property of $D_{12}$ under (6.4) is given as follows.

$$
\begin{aligned}
\left(L_{-1}^{h(1)}+L_{-1}^{h(2)}\right) D_{12}= & 0, \\
\left(L_{0}^{h(1)}+L_{0}^{h(2)}\right) D_{12}= & -(2+\Delta) D_{12} \\
\left(L_{1}^{h(1)}+L_{1}^{h(2)}\right) D_{12}= & -i\left[2\left(x_{1}^{+}+x_{2}^{+}\right)+2 i\left(\beta_{1}^{+}-\beta_{2}^{+}\right)+\Delta_{1}\left(x_{1}^{+}+x_{2}^{+}\right)\right. \\
\left(W_{-2}^{h(1)}+W_{-2}^{h(2)}\right) D_{12}= & \left.0, \quad-3 i \mu_{1}\left(\beta_{1}^{+}-\beta_{2}^{+}\right)\right] D_{12}, \\
\left(W_{-1}^{h(1)}+W_{-1}^{h(2)}\right) D_{12}= & \left.0, \quad-3 \mu_{1}\left(x_{1}^{+}-x_{2}^{+}\right)\right] D_{12}, \\
\left(W_{0}^{h(1)}+W_{0}^{h(2)}\right) D_{12}= & 0, \quad-i\left[2\left(x_{1}^{+}-x_{2}^{+}\right)+2 i\left(\beta_{1}^{+}+\beta_{2}^{+}\right)+i \Delta_{1}\left(\beta_{1}^{+}+\beta_{2}^{+}\right)\right. \\
\left(W_{1}^{h(1)}+W_{1}^{h(2)}\right) D_{12}= & \\
\left(W_{2}^{h(1)}+W_{2}^{h(2)}\right) D_{12}= & 4\left[4 i\left(\alpha_{1}^{+}+\alpha_{2}^{+}\right)-\left(i \beta_{1}^{+}+x_{1}\right)^{2}+\left(i \beta_{2}^{+}-x_{2}^{+}\right)^{2}\right. \\
& -2\left(\beta_{1}^{+}\right)^{2}+2\left(\beta_{2}^{+}\right)^{2}+2 i \Delta_{1}\left(\alpha_{1}^{+}+\alpha_{2}^{+}\right) \\
& -i \Delta_{1}\left(x_{1}^{+} \beta_{1}^{+}+x_{2}^{+} \beta_{2}^{+}\right) \\
& \left.+\frac{3}{2} \mu_{1}\left\{\left(\beta_{1}^{+}\right)^{2}-\left(\beta_{2}^{+}\right)^{2}+\left(x_{1}^{+}\right)^{2}-\left(x_{2}^{+}\right)^{2}\right\}\right] D_{12},(6.16
\end{aligned}
$$

where $\Delta_{2}=\Delta_{1}$ and $\mu_{2}=-\mu_{1}$ are used.

Let us now consider three-point function $G_{123}$ of $O_{\Delta_{i}, \mu_{i}}\left(x_{i}^{+}, \alpha_{i}^{+}, \beta_{i}^{+}\right)$'s $(i=1,2,3)$, although analysis in this paper is restricted to $c \rightarrow \infty$ limit. This is interesting in $\mathrm{W}_{3}$ CFT in its own right.

$$
\begin{aligned}
& G_{123}\left(x_{1}^{+}, \alpha_{1}^{+}, \beta_{1}^{+} ; x_{2}^{+}, \alpha_{2}^{+}, \beta_{2}^{+} ; x_{2}^{+}, \alpha_{2}^{+}, \beta_{2}^{+}\right) \\
& =\left\langle 0\left|T O_{\Delta_{1}, \mu_{1}}\left(x_{1}^{+}, \alpha_{1}^{+}, \beta_{1}^{+}\right) O_{\Delta_{2}, \mu_{2}}\left(x_{2}^{+}, \alpha_{2}^{+}, \beta_{2}^{+}\right) O_{\Delta_{3}, \mu_{3}}\left(x_{3}^{+}, \alpha_{3}^{+}, \beta_{3}^{+}\right)\right| 0\right\rangle(6.1
\end{aligned}
$$


Like the two-point function, $G_{123}$ is a solution to the following equations

$$
\left(\sum_{i=1}^{3} L_{n}^{h(i)}\right) G_{123}=0, \quad\left(\sum_{i=1}^{3} W_{n}^{h(i)}\right) G_{123}=0 .
$$

and $G_{123}$ is non-zero only for $\mu_{1}+\mu_{2}+\mu_{3}=0$. Contrary to the case of global Virasoro algebra without $\mathrm{W}$-algebra extension, there are three linearly independent solutions to these equations.

$$
\begin{gathered}
G_{123}=\left(D_{12} D_{12}^{*}\right)^{-\frac{1}{8}\left(\Delta_{1}+\Delta_{2}-\Delta_{3}\right)}\left(D_{23} D_{23}^{*}\right)^{-\frac{1}{8}\left(\Delta_{2}+\Delta_{3}-\Delta_{1}\right)} \\
\left(D_{13} D_{13}^{*}\right)^{-\frac{1}{8}\left(\Delta_{1}+\Delta_{3}-\Delta_{2}\right)} K, \\
K=\left\{\begin{array}{c}
\left(\frac{D_{13}}{D_{13}^{*}}\right)^{\frac{3}{4} \mu_{1}}\left(\frac{D_{23}}{D_{23}^{*}}\right)^{\frac{3}{4} \mu_{2}}, \text { or } \\
\left(\frac{D_{21}}{D_{21}^{*}}\right)^{\frac{3}{4} \mu_{2}}\left(\frac{D_{31}}{D_{31}^{*}}\right)^{\frac{3}{4} \mu_{3}}, \text { or } \\
\left(\frac{D_{32}}{D_{32}^{*}}\right)^{\frac{3}{4} \mu_{3}}\left(\frac{D_{12}}{D_{12}^{*}}\right)^{\frac{3}{4} \mu_{1}}
\end{array}\right.
\end{gathered}
$$

For $K$ linear combination of these terms is possible. There is, however, some restriction depending on the operators. When one of the operator is an identity, a three-point function must reduce to a two-point function. If $O_{\Delta_{3}, \mu_{3}}$ is an identity, $\mu_{3}=0$ and $\Delta_{1}=\Delta_{2}$. Then the solution in the first line of $K$ cannot be present, because the coordinates $x_{3}^{ \pm}$do not cancel.

\section{RG Flow}

In the previous section correlation functions of holomorphic quasi-primary operators at point $\left(x^{+}, \alpha^{+}, \beta^{+}\right)$with conformal weight $h=\frac{\Delta}{2}$ and $\mathrm{W}_{3}$ charge $\mu$ are computed. It is interesting whether there exists a point where the conformal weight changes, if the parameters $\beta^{ \pm}$are changed. We will study this problem by investigation of two (and three) point functions.

The two-point function (6.8) is written in terms of a function $D_{12}$ (6.9) and its complex conjugate $D_{12}^{*}$. If two operators are put on a hypersurface with $\alpha^{+}=\alpha_{0}$ and $\beta^{+}=\beta_{0}$, then two operators have $\alpha_{i}^{+}=\alpha_{0}$ and $\beta_{i}^{+}=\beta_{0}(i=1,2)$, and $\alpha_{0}$ disappears from $D_{12}$, while $\beta_{0}$ remains:

$$
D_{12} \rightarrow\left(x_{1}^{+}-x_{2}^{+}\right)^{2}+4 i\left(x_{1}^{+}-x_{2}^{+}\right) \beta_{0}=\left(x_{1}^{+}-x_{2}^{+}\right)\left[x_{1}^{+}-x_{2}^{+}+4 i \beta_{0}\right]
$$

For $\beta_{0}=0$ this corresponds to conformal two-point function with conformal dimension $\Delta_{1}$. However, the background with non-vanishing $\beta_{0}$ breaks conformal 
invariance 15 Conformal invariance is recovered by taking $\beta_{0}$ to infinity after changing the normalization of the quasi-primary field by multiplication of an appropriate power of $\beta_{0}$, i.e., $\beta_{0}^{\frac{1}{2} \Delta_{1}}$. Because the factor which contains $\beta_{0}$ cancels with this multiplication factor in the two-point function (6.8), the conformal dimension changes from $\Delta_{1}$ to $\frac{1}{2} \Delta_{1}$ and $\mathrm{W}_{3}$ charge $\mu$ vanishes at the end of the limit. Same conclusion can be reached for the three-point function (6.13). Hence the CFT flows to another one.

To summarize, operators

$$
\hat{\Phi}\left(x^{+}, \beta_{0}\right)=\beta_{0}^{\frac{1}{2} \Delta} e^{i x^{+} L_{-1}^{h}} e^{\beta_{0} W_{-1}^{h}} e^{L_{-1}} \Phi(0) e^{L_{1}} e^{-\beta_{0} W_{-1}^{h}} e^{-i x^{+} L_{-1}^{h}},
$$

where $\Phi(0)$ 's are holomorphic primary operators with $h=\frac{\Delta}{2}$ at the origin of Euclidean plane, have conformal correlation functions of conformal weight $h^{\prime}=\frac{1}{2} h$ in the limit $\beta_{0} \rightarrow \infty$. This RG flow proceeds along $\beta$ direction at $y=0$ in the higher dimensional space with metric (5.5). This is similar to the holographic RG flow in the radial direction $y$. Because $\beta^{ \pm}$appears in the metric (5.5) in the combination $\beta^{+} \beta^{-} / y^{4}$ with $y$, the limit $\beta^{ \pm} \rightarrow \infty$ corresponds to $y \rightarrow 0$, i.e. UV.

In [22] and [23] two vacua corresponding to two types of embedding of $\operatorname{sl}(2, \mathrm{R})$ algebra into sl $(3, \mathrm{R})$ are studied. One embedding, a principal one, employs $\left(L_{1}, L_{0}, L_{-1}\right)$ as generators of $\operatorname{sl}(2, \mathrm{R})$ and corresponds to the standard $W_{3}$ algebra. In the other embedding called a non-principal one, $\left(\hat{L}_{1}, \hat{L}_{0}, \hat{L}_{-1}\right)=\left(\frac{1}{4} W_{2}, \frac{1}{2} L_{0},-\frac{1}{4} W_{-2}\right)$ generates $\operatorname{sl}(2, \mathrm{R})$ and this embedding gives rise to a new algebra known as $W_{3}^{(2)}[24]$. On the gravity side an interpolating solution connecting these two vacua was found in 22. In the non-principal embedding $W_{ \pm 2}$ and $L_{0}$ correspond to the stress tensor $T$, and the other generators of $\operatorname{sl}(3, \mathrm{R})$ to a $\mathrm{U}(1)$ current $U$ and two spin $3 / 2$ currents $G_{ \pm}$. Hence the conformal weights of the currents $h$ changes to $h^{\prime}=h / 2$. Because this pattern of the change of conformal weights is the same, this $W_{3}^{(2)}$ vacuum will also be the one to which CFT will flow in our RG. Our RG flow will, however, be triggered by some additional terms in the action, which is different from those in [22].

We will now argue that these additional terms to the action are given by

$$
\begin{aligned}
\Delta S & =-i \beta^{+} W_{-1}^{h}-i \beta^{-} \bar{W}_{-1}^{h} \\
& =-\frac{i}{4} \beta^{+}\left(W_{2}+2 W_{1}-2 W_{-1}-W_{-2}\right)-\frac{i}{4} \beta^{-}\left(\bar{W}_{2}+2 \bar{W}_{1}-2 \bar{W}_{-1}-\bar{W}_{-2}\right)
\end{aligned}
$$

\footnotetext{
${ }^{15}$ Note that here we fixed $\beta^{+}=\beta_{0}$ and do not treat it as a coordinate.
} 
There are $(-i)$ 's, because $W_{-1}^{h}$ is anti-hermitian operator and its c-number counterpart is pure imaginary. In what follows only the holomorphic part is considered. Recall that under the flow an operator $\mathcal{O}(x)=e^{i x^{+} L_{-1}^{h}} \mathcal{O}(0) e^{-i x^{+} L_{-1}^{h}}$ transforms as 16

$$
\begin{aligned}
\mathcal{O}(x) & \rightarrow e^{i x^{+} L_{-1}^{h}} e^{\beta^{+} W_{-1}^{h}} \mathcal{O}(0) e^{-\beta^{+} W_{-1}^{h}} e^{-i x^{+} L_{-1}^{h}} \\
& =e^{\beta^{+} W_{-1}^{h}} e^{i x^{+} \boldsymbol{L}_{-1}^{h}} \mathcal{O}(0) e^{-i x^{+} \boldsymbol{L}_{-1}^{h}} e^{-\beta^{+} W_{-1}^{h}} \\
& \equiv e^{\beta^{+} W_{-1}^{h}} \mathcal{O}(x) e^{-\beta^{+} W_{-1}^{h}}
\end{aligned}
$$

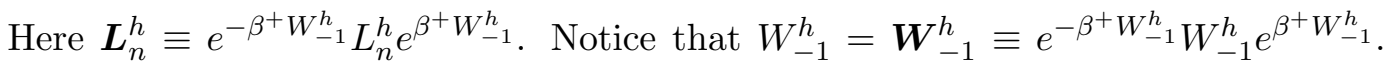
Because $L_{-1}^{h}$ and $W_{-1}^{h}$ do not commute, after the transformation by $e^{\beta^{+} W_{-1}^{h}} \operatorname{sl}(3, \mathrm{R})$ generators change. The fact that $L_{-1}^{h}$ changes to $\boldsymbol{L}_{-1}^{h}=L_{-1}^{h}-\beta^{+} W_{-2}^{h}$ is consistent with the expectation that when additional terms are added to the action, they will give rise to new contributions to the stress tensor. Furthermore for $\beta^{+} \rightarrow \infty, \boldsymbol{L}_{-1}^{h}$ corresponds to the Virasoro generator for $\mathrm{W}_{3}^{(2)}$ vacuum. $\mathcal{O}(x)$ is an abbreviation for the operator shifted by $\boldsymbol{L}_{-1}^{h}$.

Then arbitrary states of form $|\psi\rangle=\mathcal{O}_{1}\left(x_{1}^{+}\right) \mathcal{O}_{2}\left(x_{2}^{+}\right) \cdots \mathcal{O}_{n}\left(x_{n}^{+}\right)|0\rangle$ are transformed according to

$$
|\psi\rangle \rightarrow e^{\beta^{+} W_{-1}^{h}}|\psi\rangle,
$$

where $|\psi\rangle=\mathcal{O}_{1}\left(x_{1}^{+}\right) \mathcal{O}_{2}\left(x_{2}^{+}\right) \cdots \mathcal{O}_{n}\left(x_{n}^{+}\right)|0\rangle$. In the path-integral formalism this transformation is achieved by adding to the action a term $\Delta S=-i \beta^{+} W_{-1}^{h}$. As mentioned above, we regard this additional term in the action as the origin of the change of the translation generator $L_{-1}^{h}$.

In the case of a similar transformation generated by $L_{-1}^{h}$

$$
\mathcal{O}_{i} \rightarrow e^{i \delta x^{+} L_{-1}^{h}} \mathcal{O}_{i} e^{-i \delta x^{+} L_{-1}^{h}}
$$

states $|\psi\rangle$ are transformed as

$$
|\psi\rangle \rightarrow e^{i \delta x^{+} L_{-1}^{h}}|\psi\rangle=e^{i \delta x^{+}\left(H^{h}-P^{h}\right)}|\psi\rangle
$$

where $H^{h}$ and $P^{h}$ are Hamiltonian and a momentum operator, respectively. These operators translate the system along the boundary directions, but do not alter the system. There is no flow.

In the flow discussed in this paper the stress tensor $T_{++}$, which is a quasi-primary operator with $h=2$, will flow to a current $U_{+}$, which has $h=1$. This can be easily

\footnotetext{
${ }^{16}$ See (7.2). Here we changed notation and $\mathcal{O}(0)$ stands for an operator on the point $x^{ \pm}=t \pm \phi=0$ of the cylindrical boundary.
} 
shown by the same method mentioned above. Then is there a stress tensor $T_{++}^{\prime}$ at the end of flow? If the limit is also a CFT, it must exist, and this would flow from an $h=4$ current in the IR (i.e., first UV). In general there is no such quasi-primary operator in $\mathrm{W}_{3}$ extended CFT. Although there are $h=4$ operators $\Lambda$ and $\partial^{2} T$, states created by these are not quasi-primary:

$$
\begin{aligned}
L_{1}|\Lambda(0)\rangle & =5|\partial T(0)\rangle, \quad L_{0}|\Lambda(0)\rangle=4|\Lambda(0)\rangle, \\
W_{2}|\Lambda(0)\rangle & =|T(0)\rangle, \quad W_{1}|\Lambda(0)\rangle=|W(0)\rangle, \quad W_{0}|\Lambda(0)\rangle=3|W(0)\rangle \text { (7.8) } \\
L_{1}\left|\partial^{2} T(0)\right\rangle & =|\partial T(0)\rangle, \quad L_{0}\left|\partial^{2} T(0)\right\rangle=4\left|\partial^{2} T(0)\right\rangle, \\
W_{2}\left|\partial^{2} T(0)\right\rangle & =0, \quad W_{1}\left|\partial^{2} T(0)\right\rangle=|W(0)\rangle, \quad W_{0}\left|\partial^{2} T(0)\right\rangle=|\partial W(0)\rangle(7.9)
\end{aligned}
$$

Here $|T(0)\rangle \equiv \lim _{z \rightarrow 0} T(z)|0\rangle . W(z)$ and $\Lambda(z)$ are the $\mathrm{W}$ current and the normalordered product of $T, \Lambda=(T, T)-(3 / 10) \partial^{2} T$, respectively. At present , it is not clear with our method if some linear combination of $\Lambda$ and $\partial^{2} T$ would flow to the new stress tensor $T^{\prime}$. This question may be answered by constructing two-point functions which are compatible with these conditions (7.8), (7.9) and taking $\beta \rightarrow \infty$ limit. Similarly, the state created by $W$ is not also quasi-primary $\left(W_{1}|W(0)\rangle=-20|T(0)\rangle\right.$, etc.) and it is not known if two $h=3 / 2$ currents could be obtained at the end of flow. This analysis is left for future study.

\section{Summary}

In this paper 3D spin-3 gravity is studied from the point of view of bulk reconstruction and it is demonstrated explicitly that the localized state can be constructed for spin-3 gravity (in an integral form (3.9) with (3.10)). For that purpose coordinates $\alpha^{ \pm}, \beta^{ \pm}, \gamma$ of the 'internal space' which correspond to the wedge mode generators of $\mathrm{W}_{3}$ algebra, $W_{-2}, W_{-1}, W_{0}$ are introduced. The equation of motion for a scalar field is written in W space including the 'internal space' and it is found that the metric of $8 \mathrm{~d}$ gravitational background describes a $\mathrm{RG}$ flow between two $\mathrm{AdS}_{3}$ vacua. This is confirmed by calculation of two- and three-point functions of Virasoro quasi-primary operators, which are $\mathrm{W}$-descendants. By taking $\beta^{+} \rightarrow \infty$ limit, where $\beta^{+}$is a source for $W_{-1}$ generator, the two-point functions of quasi-primary fields with conformal weight $h$ flow to those with conformal weight $h^{\prime}=h / 2$. Further study of this RG flow is necessary. It is interesting to investigate how do operators $W$ and $\Lambda$ flow and whether it is possible to start with $W_{3}^{(2)}$ vacuum and then flow 
back to $W_{3}$ vacuum. In this paper it is found that by extending the spacetime to include the ' $W$ directions', $L_{n}$ and $W_{n}$ transformations acquire a meaning of diffeomorphism in the extended space in large $c$ limit, and representation of linear $\mathrm{W}_{3}$ algebra in terms of differential operators is also constructed. This construction may be extended to the modes of $L$ and $W$ outside the wedge. To summarize, in the case of $\mathrm{W}_{3}$ extended CFT, duality and holographic RG flow are realized in the extended higher-dimensional space.

It is stressed that the physical spacetime is a 3D hypersurface embedded in the higher $\mathrm{D}$ spacetime with constant $\alpha, \beta$ and $\gamma$. Its metric is given by (5.7). When these variables are varied, then the bulk and boundary theories change. When $\beta$ is changed, the boundary theory flows. $\alpha$ is a simple translation parameter. $\gamma$ can be absorbed into rescaling of $y$ as long as $\cos 2 \gamma>0$. A bulk state of a scalar field on the 3D hypersurface with fixed $\beta$ is defined in terms of a boundary state on the same hypersurface through bulk reconstruction.

Let us enumerate a few problems to study left for future. In this paper only the large $c$ limit is considered. How to incorporate $1 / c$ corrections must be studied. In this case modes outside the wedge such as $L_{n}(|n| \geq 2)$ must be taken into account due to nonlinear terms in the algebra. In the gravity background studied in this paper spin-3 gauge field is not introduced. How to define spin-3 gauge field needs to be studied. Then interpretation of the spin-3 geometry as RG flows in the $\mathrm{W}$ space may be further elucidated. It is also interesting to find and understand $\mathrm{W}_{3}$ black hole solutions in this space. 


\section{A Constraints on the Boundary State}

Transformation of $W_{n}$ in (2.2) is given by

$$
\begin{aligned}
g(\rho) W_{2} g(\rho)^{-1}= & \frac{1}{4}\left(\cosh ^{2} 2 \rho+2 \cosh \rho+1\right) W_{2}-\sinh \rho(\cosh \rho+1) W_{1} \\
& +\frac{3}{2} \sinh ^{2} 2 \rho W_{0}+W_{-1} \sinh \rho(1-\cosh \rho) \\
& +\frac{1}{4}\left(\cosh ^{2} 2 \rho-2 \cosh \rho+1\right) W_{-2}
\end{aligned}
$$

$$
\begin{aligned}
g(\rho) W_{1} g(\rho)^{-1}= & \frac{-1}{8}(\sinh 2 \rho+2 \sinh \rho) W_{2}+\frac{1}{2}(\cosh 2 \rho+\cosh \rho) W_{1}-\frac{3}{4} \sinh 2 \rho W_{0} \\
& +\frac{1}{2} W_{-1}(\cosh 2 \rho-\cosh \rho)+\frac{1}{8}(-\sinh 2 \rho-2 \sinh \rho) W_{-2},
\end{aligned}
$$

$$
\begin{aligned}
g(\rho) W_{0} g(\rho)^{-1}= & \frac{1}{8}(\cosh 2 \rho-1) W_{2}-\frac{1}{2} \sinh 2 \rho W_{1}+\frac{1}{4}(3 \cosh 2 \rho+1) W_{0} \\
& -\frac{1}{2} \sinh 2 \rho W_{-1}+\frac{1}{8}(\cosh 2 \rho-1) W_{-2}
\end{aligned}
$$

$$
\begin{aligned}
g(\rho) W_{-1} g(\rho)^{-1}= & -\frac{1}{8}(\sinh 2 \rho+2 \sinh \rho) W_{2}+\frac{1}{2}(\cosh 2 \rho-\cosh \rho) W_{1}-\frac{3}{4} \sinh 2 \rho W_{0} \\
& \left.+\frac{1}{2} W_{-1}(\cosh 2 \rho+\cosh \rho)-\frac{1}{8}(\sinh 2 \rho+2 \sinh \rho) W_{-2}, \quad \text { (A. } 4\right)
\end{aligned}
$$

$$
\begin{aligned}
g(\rho) W_{-2} g(\rho)^{-1}= & \frac{1}{4}\left(\cosh ^{2} 2 \rho-2 \cosh \rho+1\right) W_{2}+\sinh \rho(1-\cosh \rho) W_{1}+\frac{3}{2} \sinh ^{2} 2 \rho W_{0} \\
& +\frac{1}{4}\left(\cosh ^{2} 2 \rho+2 \cosh \rho+1\right) W_{-2}-\sinh \rho(\cosh \rho+1) W_{-1},
\end{aligned}
$$

Similar relations are obtained for barred (anti-holomorphic) generators.

Then the boundary limits $(\rho \rightarrow \infty)$ of $W_{n}-(-1)^{n} \bar{W}_{-n}$ are given by

$$
\begin{aligned}
& \lim _{\rho \rightarrow \infty} g(\rho)\left(W_{2}-\bar{W}_{-2}\right) g(\rho)^{-1} \\
= & \frac{1}{6} e^{2 \rho}\left(W_{2}-4 W_{1}+6 W_{0}-4 W_{-1}+W_{-2}+4 \bar{W}_{-1}-6 \bar{W}_{0}-\bar{W}_{-2}+4 \bar{W}_{1}-\bar{W}_{2}\right), \\
& \lim _{\rho \rightarrow \infty} g(\rho)\left(W_{1}+\bar{W}_{-1}\right) g(\rho)^{-1} \\
= & \frac{1}{16} e^{2 \rho}\left(-W_{2}+4 W_{1}-6 W_{0}+4 W_{-1}-W_{-2}+4 \bar{W}_{-1}-6 \bar{W}_{0}-\bar{W}_{-2}+4 \bar{W}_{1}-\bar{W}_{2}\right),
\end{aligned}
$$




$$
\begin{aligned}
& \lim _{\rho \rightarrow \infty} g(\rho)\left(W_{0}-\bar{W}_{0}\right) g(\rho)^{-1} \\
= & \frac{1}{16} e^{2 \rho}\left(W_{2}-4 W_{1}+6 W_{0}-4 W_{-1}+W_{-2}+4 \bar{W}_{-1}-6 \bar{W}_{0}-\bar{W}_{-2}+4 \bar{W}_{1}-\bar{W}_{2}\right),
\end{aligned}
$$

By using these results the following two independent conditions on the boundary state are obtained.

$$
\begin{aligned}
& \left(W_{2}-4 W_{1}+6 W_{0}-4 W_{-1}+W_{-2}\right)|\psi\rangle_{B}=0, \\
& \left(\bar{W}_{2}-4 \bar{W}_{1}+6 \bar{W}_{0}-4 \bar{W}_{-1}+\bar{W}_{-2}\right)|\psi\rangle_{B}=0 .
\end{aligned}
$$

\section{B Derivation of F (3.10)}

In this section the function $R$ in (3.10) will be obtained. Condition $\left(L_{0}^{h}-\bar{L}_{0}^{h}\right)|\psi\rangle=$ 0 is transformed to an equation on $F$ by the same procedure as that used in deriving (3.4):

$$
\left(z \partial_{z}+y \partial_{y}+2 x \partial_{x}\right) F=\left(\bar{z} \partial_{\bar{z}}+\bar{y} \partial_{\bar{y}}+2 \bar{x} \partial_{\bar{x}}\right) F .
$$

This equation implies that one of the six variables are redundant in the same way as (3.4) means $f(x, \bar{x})$ is a function only of $x \bar{x}$. Let us introduce the following five variables

$$
\zeta=z \bar{z}=|z|^{2}, \quad \xi_{1}=\frac{x}{z^{2}}, \quad \xi_{2}=\frac{y}{z}, \quad \bar{\xi}_{1}=\frac{\bar{x}}{\bar{z}^{2}}, \quad \overline{\xi_{2}}=\frac{\bar{y}}{\bar{z}} .
$$

If $F$ is regarded as a function of $\zeta, \xi_{i}$ and $\bar{\xi}_{i}$, then identities

$$
\begin{aligned}
\left(z \partial_{z}+y \partial_{y}+2 x \partial_{x}\right) F & =\zeta \partial_{\zeta} F \\
\left(\bar{z} \partial_{\bar{z}}+\bar{y} \partial_{\bar{y}}+2 \bar{x} \partial_{\bar{x}}\right) F & =\zeta \partial_{\zeta} F
\end{aligned}
$$

hold. Hence (B.1) is automatically satisfied.

Similarly, conditions from $L_{1}^{h}+\bar{L}_{-1}^{h}, W_{0}^{h}-\bar{W}_{0}^{h}, W_{1}^{h}+\bar{W}_{-1}^{h}$ and $W_{2}^{h}-\bar{W}_{-2}^{h}=0$ are given, respectively, by

$$
\begin{aligned}
& \text { - }\left[\left(y^{3}+3 y z^{2}\right) \partial_{x}-(4 x+6 y z) \partial_{y}-\left(z^{2}+3 y^{2}\right) \partial_{z}-\partial_{\bar{z}}+(\Delta-8) z\right. \\
& \left.\quad+3 \mu y+\bar{y} \partial_{\bar{x}}\right] F=0, \\
& \text { - }\left[\left(y^{2}+z^{2}\right) \partial_{x}-2 z \partial_{y}-2 y \partial_{z}-\left(\bar{y}^{2}+\bar{z}^{2}\right) \partial_{\bar{x}}+2 \bar{z} \partial_{\bar{y}}+2 \bar{y} \partial_{\bar{z}}\right] F=0, \\
& \text { - }(4 x-2 y z) \partial_{z} F-\left(y^{2}+3 z^{2}\right) \partial_{y} F+\left(2 z^{3}-4 x y\right) \partial_{x} F-\partial_{\bar{y}} F \\
& \quad+[3 \mu z+(\Delta-8) y] F=0, \\
& \text { - }\left[6 \mu\left(z^{2}-y^{2}\right)-4 \Delta y z-8 \Delta x+32 y z+64 x\right] F+\left[3 z^{4}-6 y^{2} z^{2}-y^{4}+16 x^{2}\right] \partial_{x} F \\
& \quad+\left[-4 z^{3}+12 y^{2} z+16 x y\right] \partial_{y} F+\left[4 y z^{2}+4 y^{3}+16 x z\right] \partial_{z} F+\partial_{\bar{x}} F=0 .
\end{aligned}
$$


and there are also complex conjugates of these equations. These equations are rewritten in terms of (B.2) as

- $-\left[1+3 \zeta \xi_{2}^{2}+\zeta\right] \zeta \partial_{\zeta} F+\zeta\left[\xi_{2}^{3}+3 \xi_{2}+2 \xi_{1}+6 \xi_{1} \xi_{2}^{2}\right] \partial_{\xi_{1}} F$

$$
\begin{gathered}
-\zeta\left[4 \xi_{1}+5 \xi_{2}-3 \xi_{2}^{3}\right] \partial_{\xi_{2}} F+\left(2 \bar{\xi}_{1}+\bar{\xi}_{2}\right) \partial_{\bar{\xi}_{1}} F+\bar{\xi}_{2} \partial_{\bar{\xi}_{2}} F \\
+(\Delta-8) \zeta F+3 \mu \zeta \xi_{2} F=0,
\end{gathered}
$$

• $\left[1+4 \xi_{1} \xi_{2}+\xi_{2}^{2}\right] \partial_{\xi_{1}} F-2\left[1-\xi_{2}^{2}\right] \partial_{\xi_{2}} F-2 \zeta \xi_{2} \partial_{\zeta} F$

$$
-\left[1+4 \bar{\xi}_{1} \bar{\xi}_{2}+\bar{\xi}_{2}^{2}\right] \partial_{\bar{\xi}_{1}} F+2\left[1-\bar{\xi}_{2}^{2}\right] \partial_{\bar{\xi}_{2}} F+2 \zeta \bar{\xi}_{2} \partial_{\zeta} F=0,
$$

- $\left[4 \xi_{1}-2 \xi_{2}\right] \zeta \partial_{\zeta} F+2\left[1-4 \xi_{1}^{2}\right] F+\left[-3+\xi_{2}^{2}-4 \xi_{1} \xi_{2}\right] \partial_{\xi_{2}} F-\zeta^{-1} \partial_{\bar{\xi}_{2}} F$

$$
+\left[3 \mu+(\Delta-8) \xi_{2}\right] F=0,
$$

- $\left[3-2 \xi_{2}^{2}-\xi_{2}^{4}+16 \xi_{1}^{2}-8 \xi_{1} \xi_{2}-8 \xi_{1} \xi_{2}^{3}-32 \xi_{1}^{2}\right] \partial_{\xi_{1}} F+\left[-4+12 \xi_{2}^{2}-4 \xi_{2}^{2}-4 \xi_{2}^{4}\right] \partial_{\xi_{2}} F$

$$
\begin{aligned}
& +\left[4 \zeta \xi_{2}+4 \zeta \xi_{2}^{3}+16 \zeta \xi_{1}\right] \partial_{\zeta} F+\zeta^{-2} \partial_{\bar{\xi}_{1}} F \\
& \quad+\left[6 \mu-6 \mu \xi_{2}^{2}+(32-4 \Delta)\left(\xi_{2}+2 \xi_{1}\right)\right] F=0 .
\end{aligned}
$$

Up to a multiplicative constant, solution to these equations and their complex conjugates are given by (3.10). 


\section{References}

[1] S. S. Gubser, I. R. Klebanov and A. M. Polyakov, Gauge Theory Correlators from Noncritical String Theory, Phys. Lett. B 428, 105 (1998) hep-th/9802109.

[2] M. A. Vasiliev, Equations of motion for interacting massless fields of all spins in (3+1)-dimensions, In 'Moscow 1990, Proceedings, Symmetries and algebraic structures in physics, pt 1' 15-33.

[3] A. Campoleoni, S. Fredenhagen, S. Pfenninger and S. Theisen, Asymptotic Symmetries of Three-Dimensional Gravity Coupled to Higher-Spin Fields, arXiv:1008.4744 [hep-th].

[4] M. R. Gaberdiel and R. Gopakumar, An AdS 3 Dual for Minimal Model CFTs, Phys. Rev. D83,066007 (2011) arXiv:1011.2986 [hep-th].

[5] M. R. Gaberdiel and R. Gopakumar, Triality in Minimal Model Holography, arXiv:1025.2472 [hep-th].

[6] M. Gutperle and P. Kraus, Higher Spin Black Holes, JHEP 1105, 022 (2011) arXiv:1103.4304 [hep-th].

[7] A. B. Zamolodchikov, Infinite additional symmetries in two dimensional conformal quantum field theory, Theor. Math. Phys. 65 (1985) 1205.

[8] P. Bouwknegt and K. Schoutens, W-Symmetry in Conformal Field Theory, arXiv: hep-th/9210010.

[9] A. B. Zamolodchikov, Irreversibility of the Flux of Renormalization Group in a 2D Field Theory, JETP Lett. 43 (1986) 730.

[10] A. Achucarro and P. K. Townsend, A Chern-Simons Action for ThreeDimensional anti-De Sitter Supergravity Theories, Phys. Lett. B180, 89 (1986).

[11] E. Witten, (2+1)-Dimensional Gravity as an Exactly Soluble System, Nucl. Phys. B 311, 46 (1988).

[12] M. Miyaji, T. Numasawa, N. Shiba, T. Takayanagi and K. Watanabe, c-Mera as Surface/State Correspondence in AdS/CFT, arXiv:1506.01353 [hep-th]. 
[13] K. Goto and T. Takayanagi, CFT descriptions of bulk local states in the AdS black holes, arXiv:1704.00053 [hep-th].

[14] H. Verlinde, Poking Holes in AdS/CFT: Bulk Fields from Boundary States, arXiv:1505.05069 [hep-th].

[15] Y. Nakayama and H. Ooguri, Bulk Locality and Boundary Creating Operators, arXiv:1507.04130 [hep-th].

[16] A. Hamilton, D. Kabat, G. Lifschytz, @Holographic representation of local bulk operators, Phys. Rev. D74 (2006) 066009 [arXiv: hepth/0606141].

[17] A. Hamilton, D. Kabat, G. Lyfschytz, D. A. Lowe, Local bulk operators in AdS/CFT: A Boundary view of horizons and locality, Phys. Rev. D73 (2006) 086003 [arXiv:0506118]

[18] A. Hamilton, D. Kabat, G. Lyfschytz, D. A. Lowe, Local bulk operators in AdS/CFT-A holographic description of the black hole interior, Phys. Rev. D75 (2007) [arXiv:0612053].

[19] P. Di Francesco, P. Mathieu, D. Senechal, Conformal field theory, New York, USA: Springer (1997) 890 p.

[20] V. Fateev and S. Ribault, The large central charge limit of conformal block, arXiv:1109.6764 [hep-th].

[21] P. Bowcock and G. M. T. Watts, Null vectors, 3-point and 4-point functions in conformal field theory, arXic:hep-th/9309146.

[22] P.M. Ammon, M. Gutperle, P. Kraus and E. Perlmutter, Spacetime Geometry in Higher Spin Gravity, arXiv:1106.4788 [hep-th].

[23] A. Castro, E. Hijano Cubelos, A. Lepage-Jutier and A. Maloney, , arXiv:1110.4117 [hep-th].

[24] A. Bilal, W algebras from Chern-Simons theory, Phys. Lett. B 267, 487 (1991). 